\title{
The effect of preconditioning of tailings prior to inline flocculation and deposition
}

\author{
C Torres López BASF Corporation, USA \\ M Catling BASF Corporation, USA \\ J Bellwood BASF plc, UK \\ L Boxill BASF Canada Inc., Canada
}

\begin{abstract}
Improving the dewatering characteristics of high solids tailings streams, by the addition of high molecular weight anionic polyacrylamides is known to be operationally challenging. This is particularly true in applications where a secondary flocculation occurs after primary treatment and thickening of a tailings stream - for example, underflow from a thickener or the dredging and re-treatment of unconsolidated material from a tailings dam. Previous experience has shown that high dosages of polymer are often required to increase the initial water release from the tailings on deposition and improve the longer-term consolidation of the deposit.

This paper investigates the effect of preconditioning high solids tailings through the use of shear prior to flocculation with the aim of both reducing the overall polymer dose and improving the dewatering performance of the deposit. Data presented includes the effect of preconditioning on slurry rheology and initial water release of the polymer treated material. The work showed that in some circumstances, improvements may be achieved by applying an optimised level of pre-shear, but this is dependent upon the type and properties of the tailings. This study was undertaken on tailings slurries, from different mineral types, that have varying levels of clay and overall solids content.
\end{abstract}

Keywords: pre-shear, flocculation, tailings, water release

\section{Introduction}

The mining industry generates high volumes of waste, which leads to an increase in the number of tailings storage facilities (TSF). Large areas are needed for the storage of the high quantities of waste produced, which then pose a high potential for environmental and/or public hazards (Reid et al. 2009; Walker 2015). Therefore, it is very important to employ an effective method of treatment and disposal to efficiently consolidate the tailings and have fast remediation and reclamation of the area serving as the TSF. Over the years, the use of synthetic chemicals, like flocculants, have helped towards the better disposal of tailings as they accelerate the settlement of the fine-grained particles and the tailings dewatering, making them effective at solid-liquid separation (Stocks 2006). Tailings are a mixture of different components that include sands, clays, leftover process chemicals, and large volumes of water. However, it is the fine particulate in the tailings that govern the nature of the tailings themselves. Clays are part of the fine solids fraction $(<44 \mu \mathrm{m})$ in tailings (Kotlyar et al. 1995; Masliyah et al. 2004). It is known that clays have a natural tendency to form aggregates and a high water retention capability, making this a problem when trying to dewater tails (Van Olphen 1977; Hogg 2000). This is one of the reasons why dewatering may be less efficient if the interactions between clay aggregates remain undisturbed, thus decreasing dewatering in the substrate.

One method of breaking up clay particle aggregates is using a shear force, which is the main topic investigated in this study. Consequently, shearing the slurry would interrupt interparticle interactions between the clay aggregates, releasing the trapped water and making the clay surface available for flocculation (Vajihinejad \& 
Soares 2018). Still, over-shearing may be detrimental, if it leads to an increase in the number of primary particles in the system.

For this work, two objectives were studied:

1. Investigating if levels of pre-shear prior to flocculation improves the dewatering on three different types of mine tailings, while decreasing the amount of polymer required.

2. If it was possible to overly pre-shear the slurry and whether this hindered the dewatering performance.

This was achieved by applying shear to a tailings sample for different amounts of time before treating with the flocculant. Pre-shear was applied at different time durations, which would provide insight on the amount of time necessary to achieve effective dewatering of the tailings. Free drainage dewatering was done by using sieves, then the amount of water released was quantified during the different time frames. If proven to be successful, optimal pre-shear could be used to minimise the flocculant dose needed to optimise dewatering.

\section{Methodology}

The materials used in the study and their characterisation, instruments used, and procedure followed to achieve the results are outlined in this section.

\subsection{Mine tailings samples and their characterisation}

Two different substrates were used in this study:

1. Oil sands mature fine tailings (MFT).

2. Coal thickener underflow.

The oil sands sample was obtained from Alberta, Canada and the coal thickener underflow was sourced from a coal mine in Kentucky, United States. Table 1 shows the characterisation details for each slurry while Figure 1 shows the particle size distribution graph.

Table 1 Characterisation of mine tails used in study

\begin{tabular}{lll}
\hline Tailings & Oil sands mature fine tailings & Coal thickener underflow \\
\hline Solids content $(w t \%)$ & 35.6 & 25.9 \\
\hline Methylene blue index (meq/100 g) & 8.8 & 7.5 \\
Density $(\mathrm{g} / \mathrm{mL})$ & 1.3 & 1.2 \\
Mean particle size $(\mu \mathrm{m})$ & 16.6 & 18.81 \\
$\mathrm{~d}_{10}(\mu \mathrm{m})$ & 1.8 & 2.2 \\
$\mathrm{~d}_{50}(\mu \mathrm{m})$ & 9.3 & 8.5 \\
$\mathrm{~d}_{90}(\mu \mathrm{m})$ & 38.7 & 44.5 \\
$\mathrm{pH}$ & 7.9 & 7.1 \\
\hline
\end{tabular}




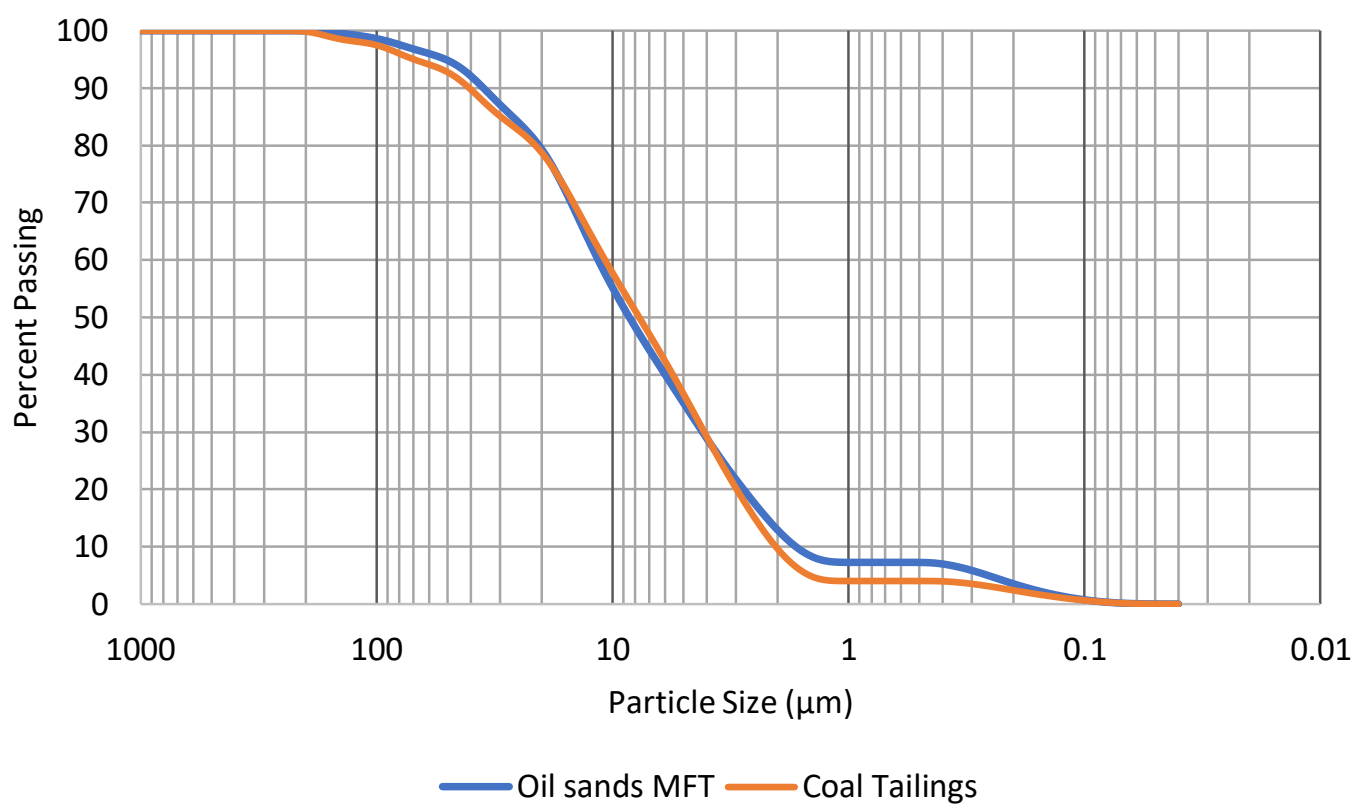

Figure 1 Particle size distribution graph for the oil sands MFT and the coal tailings

\subsection{Characteristics and preparation of polymers}

Anionic polymers from BASF were used as flocculants to treat the different mine tailings. Table 2 includes some characteristics of these polymers.

Table 2 Few characteristics of the polymers used to treat the mine tailings

\begin{tabular}{llll}
\hline Flocculant & Molecular weight & Anionic charge density (wt\%) & Substrate to treat \\
\hline Polymer A & Low-medium & 30 & Oil sands MFT \\
Polymer B & Medium & 40 & Coal thickener underflow \\
\hline
\end{tabular}

Polymer A and Polymer B were chosen to treat the oil sands MFT, and coal thickener underflow, respectively. Polymers were prepared as $0.5 \% \mathrm{w} / \mathrm{v}$ solutions from a dry polymer powder in process water (PW). Polymer $B$ was made in PW sourced from their respective mines, while Polymer A was hydrated with synthetic PW of similar chemical composition to a recycle PW sourced from an oil sands operation. The hydrated polymer solutions were initially mixed by hand for about 1 minute, then for 1 hour in a tumble wheel set at $30 \mathrm{rpm}$. Polymer A was prepared and used on the day the experiment was set to be completed, while the Polymer B solution was made the previous day before testing and spun for 2 hours on the tumble wheel.

\section{$2.3 \quad$ Instruments}

Mine tailings samples were pre-sheared using the IKA RW 20 digital and SCILOGEX OS40-Pro overhead mixers. Conditioning of the oil sands MFT and the coal thickener underflow was done with the IKA Eurostar 60 control overhead mixer. Particle size distribution data was obtained using a Beckman Coulter LS I3 320 laser diffraction particle size analyser. Yield stress data was obtained using a DV3T Brookfield rheometer. A pHTestr 30 meter was used to measure the pH of the different tailings and an OFITE model 800 8-Speed Electronic Viscometer was used to measure their viscosity.

\subsection{Experimental procedure}

A representative slurry sample of $300 \mathrm{~g}$ was measured and poured into a plastic beaker. The plastic beaker with sample was covered and its contents sheared at $500 \mathrm{rpm}$ using an overhead mixer with a flat blade 
impeller. Slurry samples were sheared at different times of 1,5 , and 60 minutes, except for the coal sample that was not pre-sheared for 1 minute. Control samples did not undergo pre-shearing.

To condition the oil sands MFT and the coal thickener underflow, an overhead mixer was used. The mixer was set at $320 \mathrm{rpm}$ and $300 \mathrm{rpm}$, respectively. The desired dose of polymer was added $\sim 1-2 \mathrm{~cm}$ above the surface of the slurry and $\sim 2 \mathrm{~cm}$ from the outside edge of the beaker. The sample was mixed until optimum structure was achieved. This was defined by the visual point of optimum net water release (NWR) observed. The beaker containing the treated slurry was removed from the overhead mixer.

Immediately after conditioning the samples, the yield stress of the treated slurry was taken using a V-72 vane for the vane method. The mass of the beaker with treated material was recorded and the sample was transferred into a $1 \mathrm{~mm}$ mesh sieve fitted with a base through a $5.1 \mathrm{~cm}$ plastic collar on top of the sieve. The beaker was scraped clean to add any remaining solids to the sample already on the sieve. The collar was removed, a stopwatch was started to record the time, and the mass of the empty beaker was recorded. The mass of the sieve with treated material, the mass of base with released water, and the yield stress (using a V-73 vane) were recorded at 10, 60, and 1,440 minutes (in the case of the oil sands MFT) and just at 60 minutes for the coal and copper thickener underflow samples. Mass values recorded were used to calculate the NWR by free drainage at each time point as shown in Equation 1:

$$
\operatorname{NWR}(\%)=\left[\frac{\left(\mathrm{V}_{\mathrm{RW}}-\mathrm{V}_{\mathrm{P}}\right)\left(\rho_{\mathrm{RW}}\right)}{\mathrm{M}_{\mathrm{S}}\left[1-\left(\frac{\mathrm{SC}_{\mathrm{S}}}{100}\right)\right]}\right](100)
$$

where:

$$
\begin{aligned}
& \mathrm{V}_{\mathrm{RW}}=\text { volume of release water in } \mathrm{mL} . \\
& \mathrm{V}_{\mathrm{P}}=\text { volume of product used in } \mathrm{mL} . \\
& \rho_{\mathrm{RW}}=\text { density of release water in } \mathrm{g} / \mathrm{mL} . \\
& \mathrm{M}_{\mathrm{S}}=\text { mass of slurry used in } \mathrm{g} . \\
& \mathrm{SC}_{\mathrm{S}}=\text { solids content of slurry in } \% .
\end{aligned}
$$

Also, values were used to calculate the solids content of the dewatered cake with Equation 2:

$$
\text { Solids content }(w t \%)=\left[\frac{\left(\frac{S_{C}}{100}\right) M_{S}}{M_{D S}}\right](100)
$$

where:

$$
\begin{aligned}
& \mathrm{SC}_{\mathrm{S}}=\text { solids content of slurry in } \% . \\
& \mathrm{M}_{\mathrm{S}}=\text { mass of slurry used in } \mathrm{g} . \\
& \mathrm{M}_{\mathrm{DS}}=\text { mass deposited on sieve in } \mathrm{g} .
\end{aligned}
$$

This procedure was repeated six times for each polymer dose at the different pre-shearing times.

\section{Oil sands mature fine tailings}

This section summaries the results found for the oil sands tailings substrate.

\subsection{Apparent viscosity profile}

Apparent viscosity was plotted as a function of the shear rate the sample was exposed to, where the different shear times tested were 0,5 , and 60 minutes, as shown in Figure 2. 


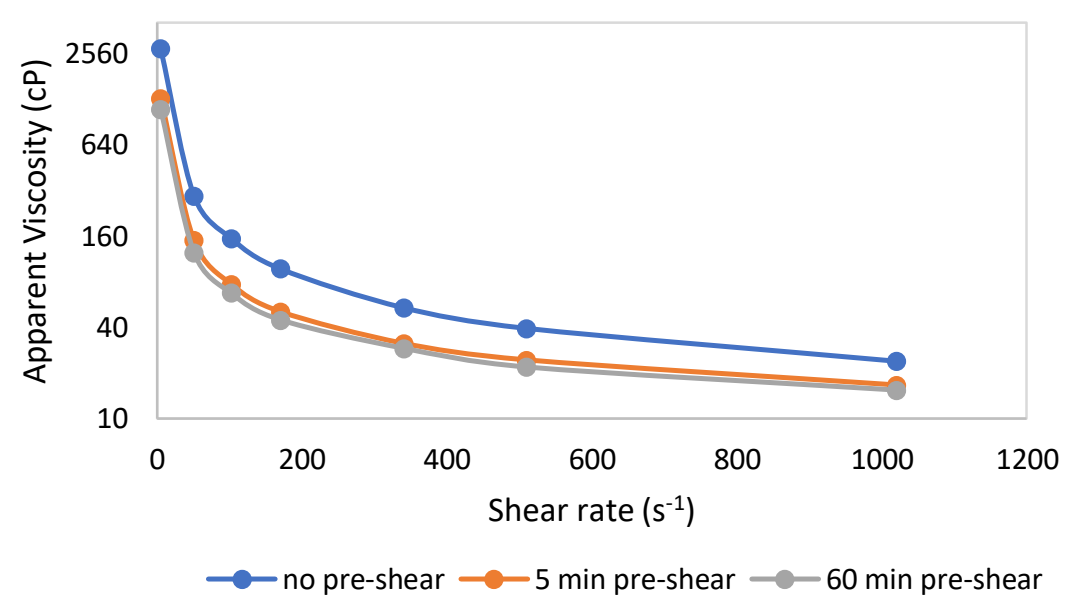

Figure 2 Apparent viscosity of the oil sands MFT at different pre-shearing times

As the MFT was exposed to prolonged periods of shear the viscosity decreased, indicating that the material was of a shear thinning nature. The interactions between the clay aggregates were disrupted when exposed to shear, accounting for the immediate drop in viscosity. Once the particles were dispersed, continued shearing of the sample then had a minor impact on its viscosity.

\subsection{Dewatering at $\mathbf{2 4}$ hours for different pre-shearing times}

Dewatering was calculated by Equation 1 and plotted at 24 hours after treating the material with Polymer $A$. The results of the free drainage dewatering of the slurry is shown in Figure 3.

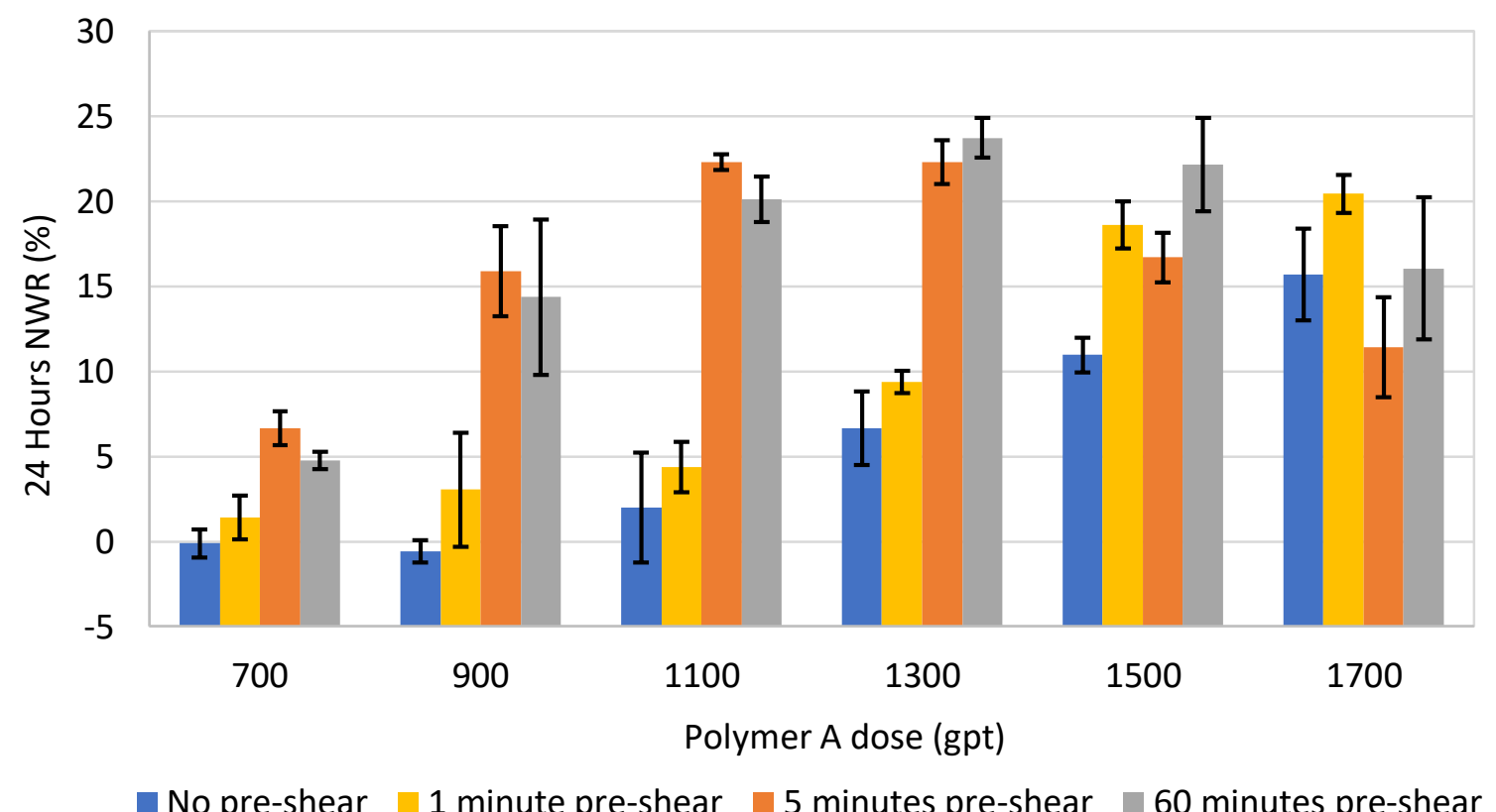

Figure 3 Net water release of the different pre-shared oil sands MFT samples after 1 hour of free drainage dewatering. Error bars are a $95 \%$ confidence error based on six tests

Limited dewatering was obtained with the MFT samples that were unsheared and pre-sheared for 1 minute, especially at low doses of polymer; nonetheless at higher doses they started to achieve substantial dewatering. Yet optimal dewatering was not achieved in either of these scenarios. Consequently, incrementally higher doses of polymer would be needed to improve dewatering. This is not a cost-effective solution for mine operators, as more polymer is needed to achieve good dewatering. 
Clay platelets tend to naturally aggregate, increasing water retention throughout the structure (Van Olphen 1977). When these aggregates are not physically dispersed, the flocculant does not interact efficiently with the particles, thus hindering the dewatering. This was seen with the sample that did not undergo pre-shear prior to treatment and the sample that was pre-sheared for just 1 minute. With the latter, even though a small increase in dewatering was observed, 1 minute was not enough time to let clay dispersion take place. This amount of shear was not enough to achieve maximum dewatering, but it proved that at least minor addition of shear before flocculation had a beneficial effect in the dewatering performance of the treated substrate.

The use of pre-shear for at least 5 minutes significantly improved the dewatering of the material, while the dose of polymer required was decreased to achieve maximum dewatering. A typical inverse parabolic relationship was seen with the dose range chosen because the underdosed, optimum dose, and overdosed scenarios were seen. The dose of 700 gpt (grams per tonne) achieved poor dewatering, meaning that the amount of polymer used was not enough to obtain optimal structure nor optimal dewatering. It reached maximum dewatering of $22.3 \%$ between 1,100 gpt and 1,300 gpt. At this amount of dewatering, the solids content of the material increased to approximately $42.6 \mathrm{wt} \%$. Dewatering decreased when overdosing because of the polymer's ability to retain water (Vedoy \& Soares 2015). NWR values for 60 minutes pre-sheared were comparable to those obtained when the MFT was pre-sheared for 5 minutes, suggesting that 'overly pre-shearing' the MFT for 1 hour did not hinder the dewatering performance of the treated substrate. The solids content of the material was comparable to the 5 minutes pre-sheared sample as it was $43 \mathrm{wt} \%$. In summary, for this oil sands substrate, at least 5 minutes of pre-shear was needed to obtain maximum amount of free water release, and a treated material with a higher solids content. As the material dewatered over time it reduced the water content present in the treated cake. Hence, the yield stress and solids content on the cake increased (Figure 4.)

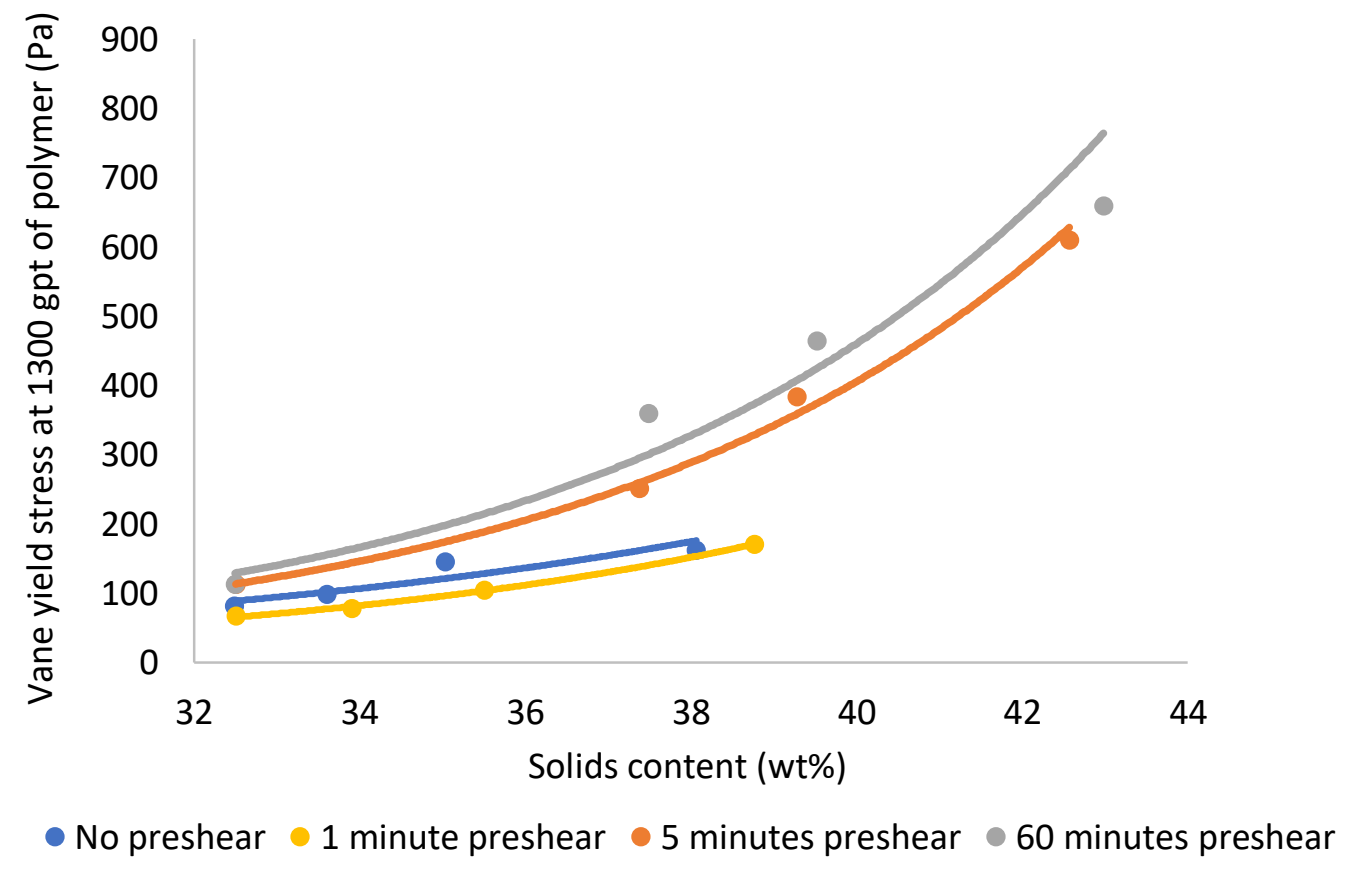

Figure 4 Vane yield stress at different stages of dewatering as a function of the solids content of the cakes pre-sheared at different times when 1,300 gpt of polymer was used

Higher solids content values were obtained for samples that achieved maximum dewatering. Fast dewatering occurred during the first 10 minutes for samples pre-sheared for 5 and 60 minutes, which resulted in a faster increase in yield stress, as the material became more plastic. In contrast, the other samples achieved similar solids content with lower yield stresses, but it was with prolonged periods of dewatering. 


\section{$4 \quad$ Coal thickener underflow}

This section summaries the results found for coal thickener underflow substrate.

\subsection{Free drainage dewatering at 1 hour}

The coal thickener underflow slurry was left to dewater for an hour as the material exhibited rapid dewatering during this time. Figure 5 shows the dewatering of the slurry at the different pre-shearing times studied throughout different doses of polymer tested.

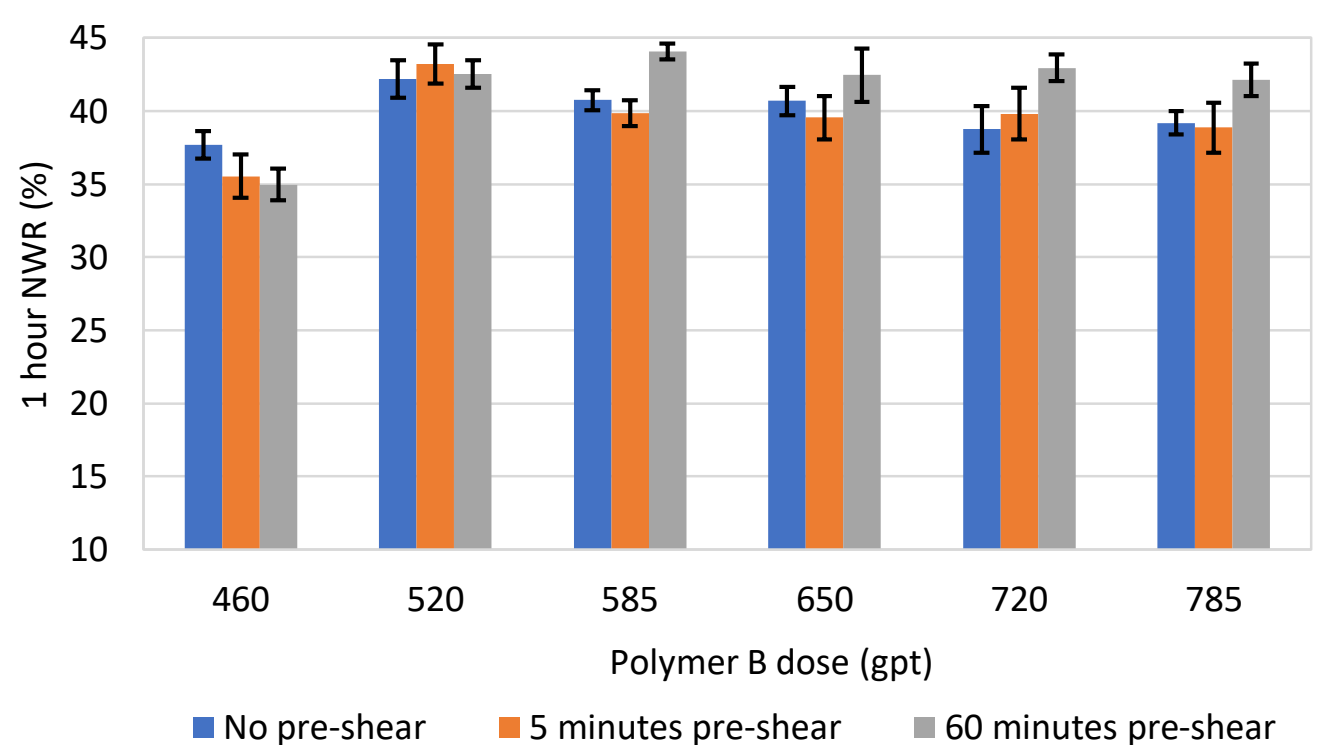

Figure 5 Net water release of the different pre-shared coal thickener underflow samples after 1 hour of free drainage dewatering. Error bars are a $95 \%$ confidence error based on six tests

There was no significant difference in dewatering between the different pre-shear times tested and the samples that did not undergo pre-shear; most of the data suggested there was no statistical difference. Dewatering was similar across the dose range tested, except for the samples that were underdosed, but still had substantial amount of dewatering when compared to the untreated coal thickener underflow. Initially, the slurry had a solids content of $25.9 \mathrm{wt} \%$, which after treatment of the optimum dose or higher doses of polymer, increased to 37-40 wt\% through the different pre-shearing times (as well as the unsheared), accounting for the dewatering of the treated slurry. Figure 6 shows a set of treated cakes undergoing dewatering when pre-shear was not applied. 


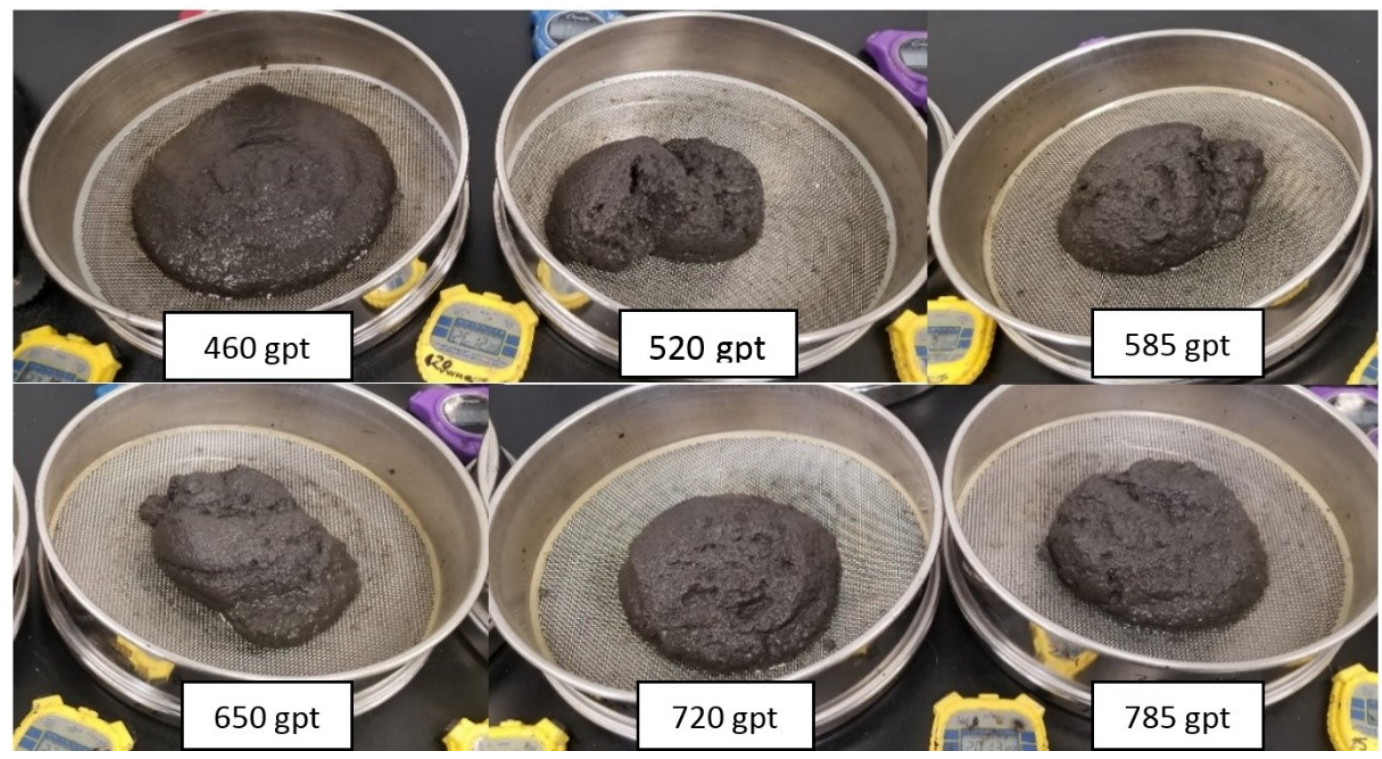

Figure 6 Examples of coal thickener underflow cakes after treatment with a specific dose of Polymer B when not exposed to pre-shear

\section{$5 \quad$ Effect of a polymer's molecular weight on dewatering}

Each of the slurries tested in this study required a different polymer for flocculation. These polymers were selected because they are currently being used by the sites to treat the respective slurries. An experiment was conducted to investigate if the molecular weight (MW) had an effect on the dewatering performance relative to the amount of shear exerted on the slurry prior to flocculation. Three different polymers with the same anionic content but different MWs were tested on the oil sands slurry. Figure 7 shows the dewatering of the different polymers tested when the slurry was unsheared and when it was pre-sheared for 5 minutes. The results showed that pre-shear was necessary to achieve a higher amount of dewatering from the sample, regardless of the polymer's MW.
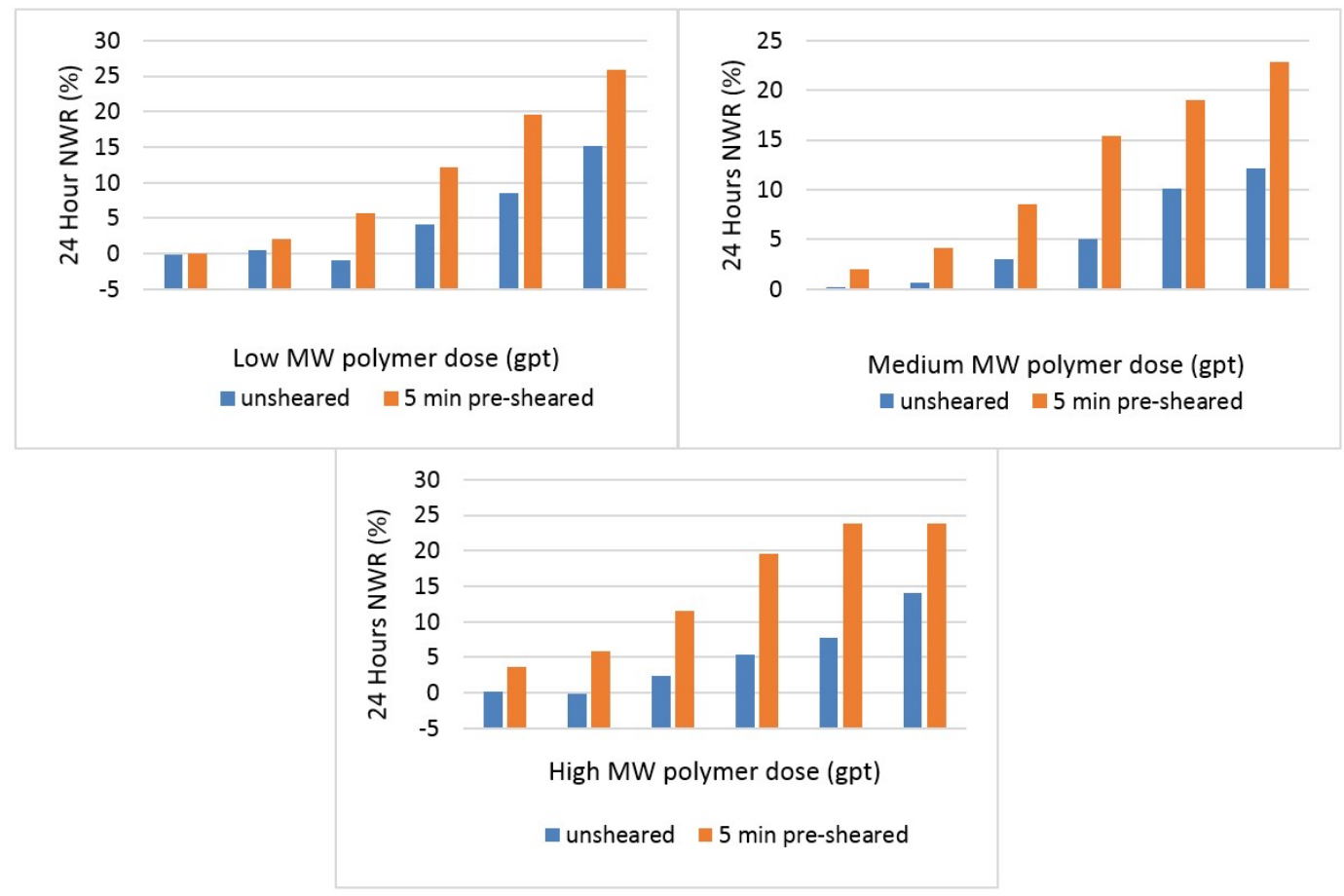

Figure 7 Net water release after 24 hours of free drainage dewatering of unsheared and 5 minutes pre-sheared oil sands mature fine tailings samples treated with different molecular weight polymers 


\section{Conclusion}

Pre-shear had different effects on each of the mine tailings tested, as it increased the dewatering performance for the oil sands MFT, decreased the dewatering observed in the copper thickener underflow, and had negligible effect on the coal thickener underflow's dewatering. The particles in the slurry must be sufficiently dispersed to enable effective interactions with the flocculant used in the treatment. Dispersion occurred in the oil sands MFT when it was pre-sheared, allowing the polymer to interact with the substrate and achieve effective dewatering of the material. Also, the results suggests that overly pre-shearing the substrate does not hinder dewatering performance for the oil sands nor for the coal slurry tested. For both substrates, applying longer periods of shear was not necessary beyond an optimal shearing point, as the particles had sufficiently dispersed across the medium. Accordingly, this saves both energy and time for the operators. The results also showed that pre-shear was necessary to obtain an increased amount of dewatering, regardless of the polymer used to treat the sample. As shown with this study, a single solution (in this case, pre-shear prior to flocculation) does not work on different types of tailings, as each have their own characteristics. Therefore, understanding the water chemistry, physico-chemical properties, rheology, or any other characteristics of the slurry is an essential part of optimising material treatment.

\section{Acknowledgement}

Thanks to BASF Corporation for granting the permission to publish this work. Also, to Carlos D. Silva Gaxiola and Ava J. Hopkinson for their hard work and support during the experimental section of the study.

\section{References}

Hogg, R 2000, 'Flocculation and dewatering', International Journal of Mineral Processing, vol. 58, pp. 223-236.

Kotlyar, LS, Sparks, BD, Woods, J, Capes, CE \& Schutte, R 1995, 'Biwetted ultrafine solids and structure formation in oil sands fine tailings', Fuel, vol. 74, no. 8, pp. 1146-1149

Masliyah, J, Zhou, ZJ, Xu, Z, Czarnecki, J, \& Hamza, H 2004, 'Understanding water-base-bitumen extraction from Athabasca oil sands', Canadian Journal of Chemical Engineering, vol. 82, pp. 628-654.

Reid, C, Bécaert, V, Aubertin, M, Rosenbaum, RK \& Deschênes, L 2009, 'Life cycle assessment of mine tailings management in Canada', Journal of Cleaner Production, vol. 17, no. 4, pp. 471-479.

Stocks, P 2006, 'Reagents', in RJ Jewell \& AB Fourie (eds), Paste and Thickened Tailings - A Guide, Australian Centre of Geomechanics, Perth.

Vajihinejad, V \& Soares, JBP 2018, 'Monitoring polymer flocculation in oil sands tailings: A population balance model approach', Chemical Engineering Journal, vol. 346, pp. 447-457.

Van Olphen, H 1977, An Introduction to Clay Colloid Chemistry, National Academy of Sciences, Washington DC.

Vedoy, DRL \& Soares, JBP 2015, 'Water-soluble polymers for oil sands tailing treatment: a review', The Canadian Journal of Chemical Engineering, vol. 93, pp. 888-904.

Walker, S 2015, 'Tailings management strategies to meet today's demands', Engineering \& Mining Journal, vol. 216, no. 11, pp. 44-49. 
